\title{
Spinning Process of Chitosan Fiber with Low Concentration of Formic Acid Solution and its Characteristics
}

\author{
Thazin Han ${ }^{1,2 *}$, Nitar Nwe ${ }^{2}$, Phyu Phyu Win ${ }^{1}$, Hiroshi Tamura ${ }^{2}$ \\ ${ }^{1}$ Department of Research and Innovation, Ministry of Education, Republic of Union of Myanmar \\ ${ }^{2}$ Faculty of Chemistry, Materials and Bioengineering and HRC, Kansai University, Suita, \\ Osaka 564-8680, Japan \\ * Corresponding author email: thazinahann@gmail.com
}

Received: 18 June 2016 / Revised: 19 July 2016 / Accepted: 20 July 2016 / Published: 27 July 2016

\begin{abstract}
The preparation of chitosan fiber was approached to spin method. The wet spinning of chitosan fiber was carried out using $7 \%$ chitosan concentration, $4 \%$ aqueous formic acid as a solvent for chitosan and $6 \mathrm{M}$ of aqueous $\mathrm{CaCl}_{2} \cdot 2 \mathrm{H}_{2} \mathrm{O}$ as a coagulation system. A better method for preparation of chitosan spinning solution was investigated by studying the effect of reaction time on incubation of spinning solution in open air. The shear viscosity of chitosan solution (22.63 23.09 Pa.s) was found to be stabilize the spinning of chitosan fiber in this study. The characteristics of different chitosan fibers were determined by FTIR and ${ }^{1} \mathrm{HNMR}$ spectroscopies, XRD diffraction, scanning electron microscopy (SEM) and mechanical properties. All the fibers were observed with high tenacity (dTex). The strength of fiber and water retention of chitosan fiber (\%) was significantly increased with increasing the incubation time of spinning solution in open air.
\end{abstract}

Keywords: aqueous formic acid; chitosan; fiber; tenacity; water retention.

\section{Introduction}

Chitin is a natural polymer of the second most abundance in the world and linked with poly (14) 2 -acetamido-2deoxy- $\beta$-D glucose and poly (14) 2 -amino-2deoxy- $\beta$-D glucose. Chitin and its derivative, chitosan can be obtained from cell wall of some fungus and yeast, the shells of shrimp, crab and the exoskeleton of insects [1]. Chitosan can be generally prepared from the Ndeacetylation of chitin. Due to chitosan molecule with free amino (NH 3+) groups and hydroxyl $(\mathrm{OH}-)$ groups on its backbone; it can be easily modified by means of chemical reactions [2]. Chitosan as a kind of copolymer is readily transformed into fibers, films, beads and scaffolds, and those kinds of materials are more recently applied in food packaging, in pharmaceutical fields, biomedical materials, textile and tissue engineering researches [3]- [5]. Chitosan based materials are biodegradable, nontoxic and biocompatible [6]. Chitosan can properly dissolve in almost dilute acidic acid solution at specific conditions such as acetic acid, citric acid, phosphoric acid, hydrochloric acid, lactic acid, malic acid, succinic acid, formic acid, etc [7] [8]. When chitosan dissolves in acid solution, it has own electro conductivity in each acid solution [9]. 
Spinning Process of Chitosan Fiber with Low Concentration of Formic Acid Solution and its Characteristics

The most common method used for preparation of chitosan fiber is wet spinning method by using suitable coagulation solutions [10]. The most influenced parts of chitosan fiber formation are spinning solution, coagulation system and washing system [11][12]. There have been several studies for chitosan fiber as chitosan filament; the first report for it was developed from Mitsubishi Rayon Co., Ltd [13]. The processes were involved with the aqueous acetic acid as solvent system, then aqueous $\mathrm{NaOH}$ and sodium lauryl sulphate as coagulants [14]. Further attempt for chitosan fiber was reported that the chitosan fiber could be produced with aqueous di-chloroacetic acid as the solvent system and $\mathrm{CuCO}_{3}-\mathrm{NH}_{4} \mathrm{OH}$ as the coagulant [15]. There has been a patent from United States; it has been stated the manufacturing of chitosan fiber; comprising the steps of dissolving chitosan having a degree of deacetylation, not less than 60\%, in an aqueous solution of sodium thiocyanate having a concentration of not less than $44 \%$ by weight, and molding the resulting spinning solution by wet spinning to form a chitosan fiber [16]. Later on, chitosan powder was used to the modification of fiber as the chitosan coated alginate filament where the small amount of chitosan was used in the first coagulation bath [17]. Chitosan spotted alginate fiber has also been developed by wet spinning of alginate solutions containing emulsified chitosan-citrate complex and studied the characteristic of those fibers [18]. The carboxy methyl chitosan with alginate has also been prepared and found some improvements compared to the pure alginate fiber [19]. It had also been studied the alginate/chitosan hybrid fiber scaffolds for tissue engineering [20] and the formation of cross-linked chitosan fiber by epichorohydrin as a cross-linking agent in a wet spinning system for the improvement of chitosan fiber [21].

Most of the previous works on the chitosan fiber have been studied with an aqueous acetic acid (1$10 \%$ by volume glacial acetic acid in water) as a solvent system and some organic solvents in the coagulation system but the basic data on the chitosan fiber as mechanical properties and crystallinity are not affected on the solvent system of the chitosan spinning solution. However, the mechanical properties of chitosan fiber have been affected on the chelating agents, washing system and drying system of that fiber. The best coagulation system and washing system of chitosan fiber have already published in our previous study [22]. The main objective of present study was the development of new method of chitosan for spinning by applying aqueous formic acid as the solvent of chitosan spinning solution, then modifying the coagulation system and washing system of chitosan fiber. This study was also carried out on some interactions of chitosan molecule with formic acid in open air and this spinning solution may affect the fiber formation and its characteristics. It has also been emphasized on water retention and antimicrobial activity of the resulted chitosan fibers.

\section{Materials and Methods}

\subsection{Materials and Reagents}

Crab chitosan (CTS) with medium molecular weight $(\mathrm{Mw}=2 \mathrm{x} 105 \mathrm{Da}, \mathrm{Mn}=1 \mathrm{x} 105 \mathrm{Da})$, the degree of deacetylation $80 \%$ (DD \%) was obtained from Koyo Chemical Co., Ltd., but further purification and modification were not done for this study. Other materials and chemicals were purchased from Wako pure chemical Co., Ltd. Japan.

\subsection{Preparation of Chitosan Solution for Spinning}

Chitosan solution ( $7 \%$ weight/volume, w/v) were prepared by dissolving $7 \mathrm{~g}$ chitosan powder in $100 \mathrm{ml}$ of $4 \%$ aqueous formic acid solution under vigorous stirring and then incubated in open air by covering the solution with a thin nylon net sheet at room temperature for 0, 2 and 4 weeks (Denoted as 0 wk CTS, 2 wk CTS and 4 wk CTS). After incubation, chitosan solutions were adjusted to obtain the same volume as original solution using 4\% aqueous formic acid solution and 15\% ethyl acetate; it was added as a lubricant to enhance spin ability as per Table 1. The chitosan solutions before and after adjusting the volume were measured the viscosity using HAAKE RheoStress 600 Rheometer at $25{ }^{\circ} \mathrm{C}$. Average shear viscosity measurement of each 
Han et al., J. Mod. Mater.; Vol. 1, Issue 1, pp: 24-34, 2016

chitosan solution was carried out for five independent replicates.

Table 1: Shear viscosity of chitosan solution $(100 \mathrm{ml})$

\begin{tabular}{|c|c|c|c|c|c|}
\hline \multirow{2}{*}{$\begin{array}{l}\text { Chitosan } \\
\text { solution } \\
\text { for fiber } \\
\text { spinning }\end{array}$} & \multirow{2}{*}{$\begin{array}{c}\text { Incuba } \\
\text { tion } \\
\text { period } \\
\text { (week) }\end{array}$} & \multicolumn{2}{|c|}{$\begin{array}{c}\text { Volume } \\
\text { adjustment }\end{array}$} & \multirow[t]{2}{*}{$* \mathbf{A}$} & \multirow[t]{2}{*}{$* \mathbf{B}$} \\
\hline & & $\begin{array}{c}\text { Ethyl } \\
\text { Acetate } \\
\text { (ml) }\end{array}$ & $\begin{array}{c}4 \% \\
\text { Formic } \\
\text { Acid } \\
\text { (ml) }\end{array}$ & & \\
\hline $\begin{array}{l}0 \mathrm{wk} \\
\text { CTS }\end{array}$ & 0 & 0 & 0 & $\begin{array}{l}23.09 \\
\pm 0.97\end{array}$ & $\begin{array}{l}23.09 \\
\pm 0.97\end{array}$ \\
\hline $\begin{array}{l}2 \mathrm{wk} \\
\text { CTS }\end{array}$ & 2 & 15 & 11 & $\begin{array}{r}31.79 \\
\pm 2.42\end{array}$ & $\begin{array}{l}22.63 \\
\pm 2.5\end{array}$ \\
\hline $\begin{array}{l}4 \mathrm{wk} \\
\text { CTS }\end{array}$ & 4 & 15 & 22.5 & $\begin{array}{c}42.6 \pm \\
2.8\end{array}$ & $\begin{array}{l}23.74 \\
\pm 0.6\end{array}$ \\
\hline
\end{tabular}

$A=$ Shear viscosity of chitosan solution before volume adjustment (Pa.s)

$B=$ Shear viscosity of chitosan solution after volume adjustment (Pa.s)

*The shear viscosity was observed at the shear rate of $100 \mathrm{~s}^{-1}$. Data were observed as mean \pm standard deviation $(n=3)$.

\subsection{Spinning of the Chitosan Fiber}

The chitosan solutions (B, Table 1) were placed in spinning columns and then removed the air bubbles from chitosan solutions. The wet spinning process was carried out at the room temperature under pressure $\left(0.6-0.8 \mathrm{Kgf} / \mathrm{cm}^{-2}\right)$ to inject the chitosan solution through 30 holes (diameter of which was $0.2 \mathrm{~mm}$ ) into the coagulation bath containing the $6 \mathrm{M} \mathrm{CaCl}_{2} \cdot 2 \mathrm{H}_{2} \mathrm{O}$ solution. The resulted yarns 30 individual fibers were then drawn between two sets of rollers with the stretching ratio of $1.0-1.2$ and then chitosan fibers were collected with bobbins. The fibers on the cassette were washed with $5 \mathrm{M} \mathrm{NaOH}$ : ethanol (2:1) solution that was used to remove the formate and calcium ions on and in the fiber, washed several times with distilled water and then washed one time with ethanol at room temperature. Each single fiber was split up from the bobbins under wet condition. Finally, fibers were dried under open air at room temperature for their characterization.

\subsection{Characteristics of the Chitosan Fiber}

The molecular structures of CTS powder and CTS fibers were confirmed by FTIR and ${ }^{1} \mathrm{HNMR}$ spectroscopies. The IR spectrum was investigated with a Shimadzu FTIR-2100 (PerkinElmer) spectrometer scanning at $400-4000 \mathrm{~cm}^{-1}$ using $\mathrm{KBr}$ method. The sample disc was measured after placing in oven at $60^{\circ} \mathrm{C}$ for $6 \mathrm{~h}$ to remove the moisture and then placed in the vacuum chamber for $3 \mathrm{~h}$. The chitosan powder and fibers were firstly dissolved in $1 \% \mathrm{D}_{3} \mathrm{COOD}$ with $\mathrm{D}_{2} \mathrm{O}$ to obtain $0.5 \%$ chitosan solution that was analyzed by ${ }^{1} \mathrm{HNMR}$ spectroscopy using ECA $400 \mathrm{MHz}$ instrument. Each fiber sample was coated with thin layer of platinum, using a JOLE JFC-1500 sputtering device and observed under SEM (FE-SEM JEOL JSM-6700 scanning electron microscope) at an accelerating voltage of $5 \mathrm{kV}$ and current of $10 \mu \mathrm{A}$. The diameter of fiber in each sample was measured using the JEOLImage software. To observe the cross-section of chitosan fiber, each fiber was cut using blade. Determination of the crystallinity of chitosan fiber was done by X-ray diffraction pattern that was recorded by using a Rigaku (R-AXIS RAPID II) diffractometer with scanning scope of $0-40^{\circ}$, scanning speed of $4^{\circ} / \mathrm{min}$, using nickel-filtered $\mathrm{Cu} \mathrm{K} \alpha$ radiation. The $\mathrm{X}$-rays were generated at 40 $\mathrm{kV}$ and $100 \mathrm{~mA}$ for pattern of samples. The $2 \theta$ (deg) of each peak in the range $0-40^{\circ}$ on the equatorial peak and the meridian of these fibers was measured for $180 \mathrm{sec}$. Calcium content in and on chitosan fiber was measured by Shimadzu ICPS 7510 sequential plasma spectrometer after the chitosan fiber was digested with nitric acid $(63 \%)$ and hydrogen peroxide at $110^{\circ} \mathrm{C}$ for $3 \mathrm{~h}$.

\subsection{Mechanical Properties of Chitosan Fiber}

Each chitosan fiber was sandwiched between two pieces of sandpaper on either side of it with the help of Scotch permanent adhesive glue. About $20 \mathrm{~mm}$ of fiber was glued on either side to the middle of the paper pieces. Mechanical properties of chitosan fibers in wet and dry states were evaluated on a universal machine (an ORIENTEC STA-1150 RTC) with $50 \mathrm{~N}$ load cell, using a cross head speed of 10 mm.min-1 and a gauge length (uniformly reduced section) between the two grips of $10 \mathrm{~mm}$. Sandpaper were attached to both sides of the grips. The end of each grip was the beginning of the attached sample in order to prevent the deformation of 
Spinning Process of Chitosan Fiber with Low Concentration of Formic Acid Solution and its Characteristics

samples during mounting. According to the standard method, the temperature was kept at 25 ${ }^{\circ} \mathrm{C}$ and all the data were instantaneously recorded with a computer. Average value from 6 replicates of each sample was recorded the tensile strength, maximum strain, elongation percent and young modulus.

\subsection{Water Retention of the Chitosan Fiber}

Each dried chitosan fiber sample was weighed and dipped into the distilled water for $24 \mathrm{~h}$ at room temperature. The water retention values (WRV) of chitosan fibers were determined at room temperature and calculated as equation 1 ;

$\mathrm{WRV}=\left(\mathrm{W}_{1}-\mathrm{W}_{0}\right) / \mathrm{W}_{0} \times 100 \%$

Where $\mathrm{W}_{0}$ represents as the original weight $(\mathrm{g})$ of each fiber sample which dried at $105^{\circ} \mathrm{C}$ for $24 \mathrm{~h}$ to obtain the constant weight, $\mathrm{W}_{1}$ is the weight of fiber sample that was centrifuged the water absorbed fibers at $4500 \mathrm{rpm}$ for 10 minutes.

\subsection{Antimicrobial Activity of Chitosan Fiber}

Escherichia coli W3110 and Staphylococcus aureus 209P for antimicrobial assay were kindly provided by Prof. Tetsuaki Tsuchido from Department of Fermentation Technology, Faculty of Chemistry, Materials and Bioengineering, and HRC, Kansai University, Osaka, Japan. E. coli and S.aureus were inoculated on Luria-Bertani (LB) Agar (Polypepton 1\%, Yeast extract $0.5 \%, \mathrm{NaCl} 1 \%$, and $1.5 \%$ agar, $\mathrm{pH}$ 7.0-7.4, from DAIGO, Nihon Seiyaku) plates and then incubated at $370 \mathrm{C}$ for overnight. One loop of each bacterium was inoculated to each sterilized culture test tube containing $5 \mathrm{ml}$ of LB broth medium in the shaking incubator at 370C for overnight. The resultant culture has 109 bacteria/ml of culture medium. For sample preparation, the fiber samples were placed in the test tube containing $5 \mathrm{ml}$ of $\mathrm{LB}$ broth and sterilized the sample tubes at $1210 \mathrm{C}$ for 15 minutes. Each sample tube was inoculated with $100 \mu \mathrm{l}$ of seed culture and incubated in a shaking incubator at $370 \mathrm{C}, 200 \mathrm{rpm}$ for $24 \mathrm{~h}$. The turbidity of the samples was measured at $0,6,12$, 18, 24 h incubation using UV spectrophotometer at $660 \mathrm{~nm}$. The number of bacteria was calculated using standard curve (OD660 Vs. No. of bacteria $/ \mathrm{ml}$ ).

\section{Results and Discussion}

\subsection{Preparation of Chitosan Fiber}

In our previous study, chitosan filament had been spun with $10 \%$ chitosan concentration in $10 \%$ aqueous acetic acid and the coagulation system was applying saturated $\mathrm{CaCl}_{2} \cdot 2 \mathrm{H}_{2} \mathrm{O}$ in methanol solution [23]-[25]. Various concentrations were prepared and tested the ability of their fiber formation in $6 \mathrm{M} \mathrm{CaCl} 2.2 \mathrm{H}_{2} \mathrm{O}$ solution. The chitosan concentration for spinning solution found to obtain a good fiber extruded into the coagulation bath. The chitosan solution concentration lower than $7 \%$ could not be produced to form fine fiber and higher than $7 \%$ chitosan solution was impossible to extrude the fiber to the coagulation bath due to high viscous in spinning solution as given in Table 2.

Table 2: Shear viscosity of different concentrations of chitosan spinning solutions

\begin{tabular}{|c|c|c|}
\hline Sample & $\begin{array}{c}\text { Chitosan } \\
\text { concentration } \\
\%(\mathbf{w} / \mathbf{v})^{*}\end{array}$ & $\begin{array}{c}\text { Shear viscosity } \\
\text { ( Pa.s)** }\end{array}$ \\
\hline 1 & 4 & $5.92 \pm 0.42$ \\
\hline 2 & 5 & $12.10 \pm 0.79$ \\
\hline 3 & 6 & $23.09 \pm 0.97$ \\
\hline 4 & 7 & $31.19 \pm 2.62$ \\
\hline 5 & 8 & $33.64 \pm 1.76$ \\
\hline 6 & 9 & $46.72 \pm 2.8$ \\
\hline 7 & 10 & \\
\hline \multicolumn{2}{|c|}{10.44} \\
*Each chitosan dissolved in $4 \%$ aqueous formic acid solution. \\
**The shear viscosity was observed at the shear rate of $100 \mathrm{~s}^{-1}$.
\end{tabular}

According to these results, it was judged that chitosan of concentration $7 \%$ was preferable for fiber formation with the aqueous formic acid as solvent system for spinning solution. Therefore, in this research, chitosan solution $7 \%(\mathrm{w} / \mathrm{v})$ was prepared and incubated in open air. In comparison, it was found to be the same concentration of chitosan (7\%) in $4 \%$ aqueous 
Han et al., J. Mod. Mater.; Vol. 1, Issue 1, pp: 24-34, 2016

acetic acid could not spin under same coagulation system. The long-time chitosan spinning solution in open air was observed the reduction of spinning solution volume, consequently an increasing shear viscosity and it was difficult to spin for chitosan fibers. Due to this fact, ethyl acetate was carried as lubricant of the spinning solution. Therefore, the chitosan solutions before and after adjusting the volume with $4 \%$ aqueous formic acid solution and $15 \%$ ethyl acetate were measured the shear viscosity to get the stable spinning condition. The results are summarized in Table 1. It was found that shear viscosity is one of the important factors for spinning condition and the best shear viscosity for the chitosan spinning in this study was about $22.63 \sim 23.09$ Pa.s. Due to the interactions between the chitosan molecule and carboxylic group of formic acid in the open air at time interval, the nature of spinning solutions was resulted as different conditions. Chitosan in aqueous formic acid can protonate as $\mathrm{NH}_{3}{ }^{+}$and formic acid can be as nature of carboxylic group as $\mathrm{HCOO}^{-}$, see in equation 2 .

$$
\mathrm{HCOOH}=\left[\mathrm{HCOO}_{-}^{-}\right]+\left[\mathrm{H}^{+}\right]
$$

The nature of formic acid and acetic acid in water may be carboxylic acid solvent system; some interactions could form between the chitosan molecules and formic acid in the spinning solution. Due to the electro conductivity of chitosan in aqueous formic acid higher than chitosan in aqueous acetic acid [2], the ionic interactions between amino groups of chitosan molecules and carboxylic groups of formic acid might decrease more than that of chitosan in acetic acid solution. On the other hand, the free amino groups of chitosan in the formic acid solution are more than in that of acetic acid solution. The fiber formation is occurred by the chelating of calcium ions with cross-link of the free $\mathrm{NH}_{3}{ }^{+}$group at the chitosan molecules after the chitosan solution extruded by passing through coagulation bath containing the aqueous $6 \mathrm{M} \mathrm{CaCl}_{2} \cdot 2 \mathrm{H}_{2} \mathrm{O}$ solution.

Fiber quality is depended on the washing system of the fibers. The original fibers were soluble in water due to remaining of the formate groups in the fiber. The previous study has reported the effect of washing system upon chitosan filaments; $\mathrm{NaOH}$ was the best for washing the chitosan filament to get good quality of fiber [22]. Herein washing solution, $\mathrm{NaHPO}_{4}$ in methanol (1:1) and $5 \mathrm{M} \mathrm{NaOH}$ in ethanol (2:1) had been selected for this study. When washing the fiber with $\mathrm{NaHPO}_{4}$, the resulted fiber strength was very low and SEM morphology also showed very rough surface. Therefore, $5 \mathrm{M} \mathrm{NaOH}$ : ethanol (2:1) was selected for washing of the chitosan fibers to remove the calcium ions. After washing, the chitosan fibers were stable in water and its surface was very smooth, which means that washing system was totally effective for the elimination of excess formate and calcium ion from the fiber. Figure 1 shows the figure of spinned fiber and wet fiber after dialysis.

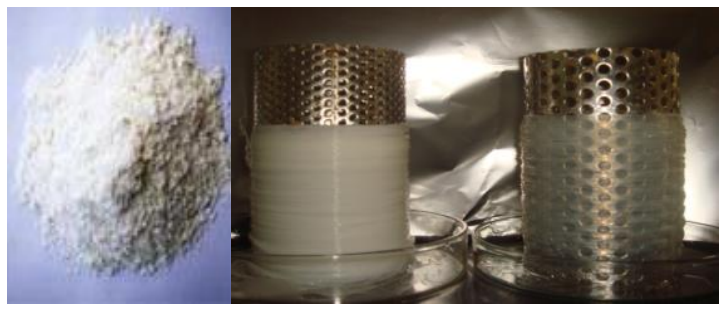

(a)

(b)

Figure 1: (a) Powder of Chitosan and (b) Spanned fiber before/after dialysis

Table 3: Calcium content of chitosan fibers after incubation of chitosan solution in open air

\begin{tabular}{|l|c|l|}
\hline $\begin{array}{l}\text { Sample of } \\
\text { fiber }\end{array}$ & $\begin{array}{l}\text { Calcium } \\
\text { content } \\
\text { mg/g of fiber) }\end{array}$ & $\begin{array}{l}\text { WRV (\%) } \\
* *\end{array}$ \\
\hline $\begin{array}{l}\text { Original CTS } \\
\text { fiber* }\end{array}$ & $20.79 \pm 1.2$ & Not detect \\
\hline 0 wk CTS & $0.0127 \pm 0.002$ & $138 \pm 6.9$ \\
\hline 2 wk CTS & $0.0152 \pm 0.0006$ & $169 \pm 6.7$ \\
\hline 4 wk CTS & $0.073 \pm 0.028$ & $333 \pm 36$ \\
\hline * The chitosan fiber was determined without washing calcium \\
from the fiber. \\
\multicolumn{3}{|c|}{$* *$ Water retention volume of CTS fibers. } \\
\multicolumn{2}{|c|}{ Data were measured as mean \pm standard deviation $(n=3)}$.
\end{tabular}

Table 3 shows the calcium content in the chitosan fibers before and after washing: The fiber before washing contained calcium ion $(20.79 \mathrm{mg} / \mathrm{g}$ of fiber) and the 0,2 and 4 wk CTS fibers after washing contained calcium ion $0.0127,0.0152$, 
Spinning Process of Chitosan Fiber with Low Concentration of Formic Acid Solution and its Characteristics

$0.073 \mathrm{mg} / \mathrm{g}$ of fiber respectively. These data suggested that the calcium ion in all fibers could be eliminated nearly equal to zero percent by $\mathrm{NaOH}$ ethanol washing system. Although the $\mathrm{NaOH}$ 's concentration was high, it could be easily washed with distilled water to reach neutrality of fiber. Washing with water only after spinning caused the adhesion of fibers. To prevent this, final washing has been achieved by using ethanol in this study.

\subsection{Characteristics of Chitosan Fiber}

Original chitosan powder and resulted fibers were analyzed by the infra-red spectrometry method to investigate the molecular structure of chitosan in fiber and shown in Figure 2. Firstly, the main absorption bands of chitosan in the spectra were clearly identified and then other bands for the modification of chitosan were studied [26].

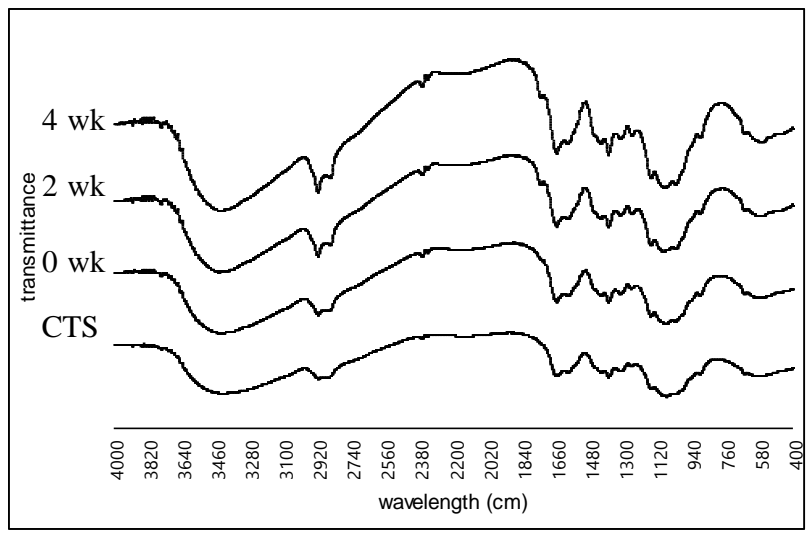

Figure 2: FTIR spectra of chitosan (CTS) powder and chitosan fibers after incubation of chitosan solution at 0, 2 and 4 weeks in open air ( 0 wk CTS, 2 wk CTS and 4 wk CTS)

The FTIR spectra of 0 wk CTS, 2 wk CTS and 4 wk CTS fibers were almost similar to the spectrum of chitosan (CTS) powder. The peaks of amide $-\mathrm{C}=\mathrm{O}$ stretching vibration at $1652 \mathrm{~cm}^{-}$ 1 , the $-\mathrm{NH}_{2}$ group bending absorption at 1592 $\mathrm{cm}^{-1},-\mathrm{CH}_{3}$ bending absorption at $1380 \mathrm{~cm}^{-1}$ significantly appeared in the FTIR spectra of chitosan power and all fibers. The hydroxyl $\mathrm{OH}^{-}$ and $\mathrm{C}-\mathrm{H}$ groups have been well identified within the wavelength number $4000-2000 \mathrm{~cm}^{-1}$ together with the absorption bands of $1652 \mathrm{~cm}^{-1}$ and 1592 $\mathrm{cm}^{-1}$ in all spectra of chitosan samples, these bands are also identification of chitosan [27]-[31] .The peak with respect to the deamination peak could not be found and any modification peaks were not observed in the spectra of all chitosan fibers. This result showed that washing system is completed after spinning. Moreover, the molecular structure of chitosan in fibers was also evaluated by using ${ }^{1} \mathrm{H}$ NMR spectroscopy and the spectra are shown in Figure 3.
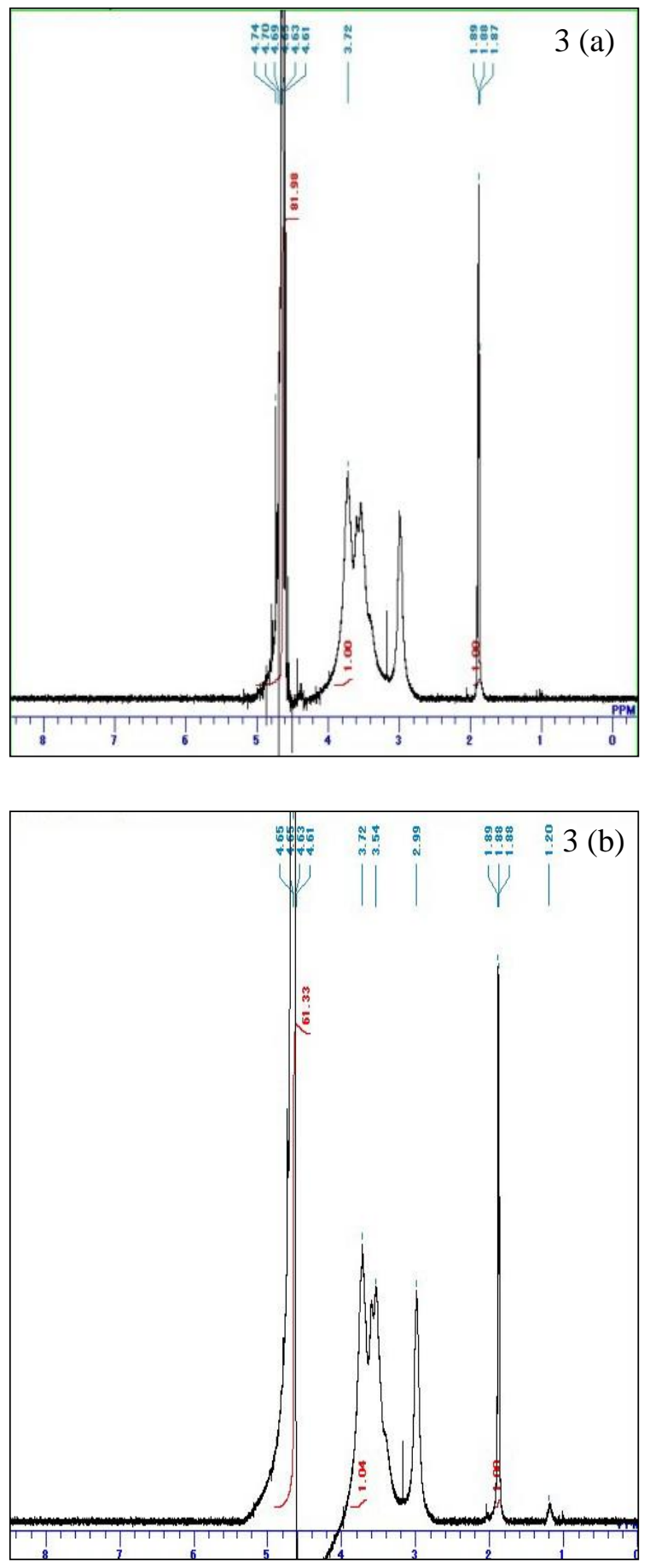
Han et al., J. Mod. Mater.; Vol. 1, Issue 1, pp: 24-34, 2016

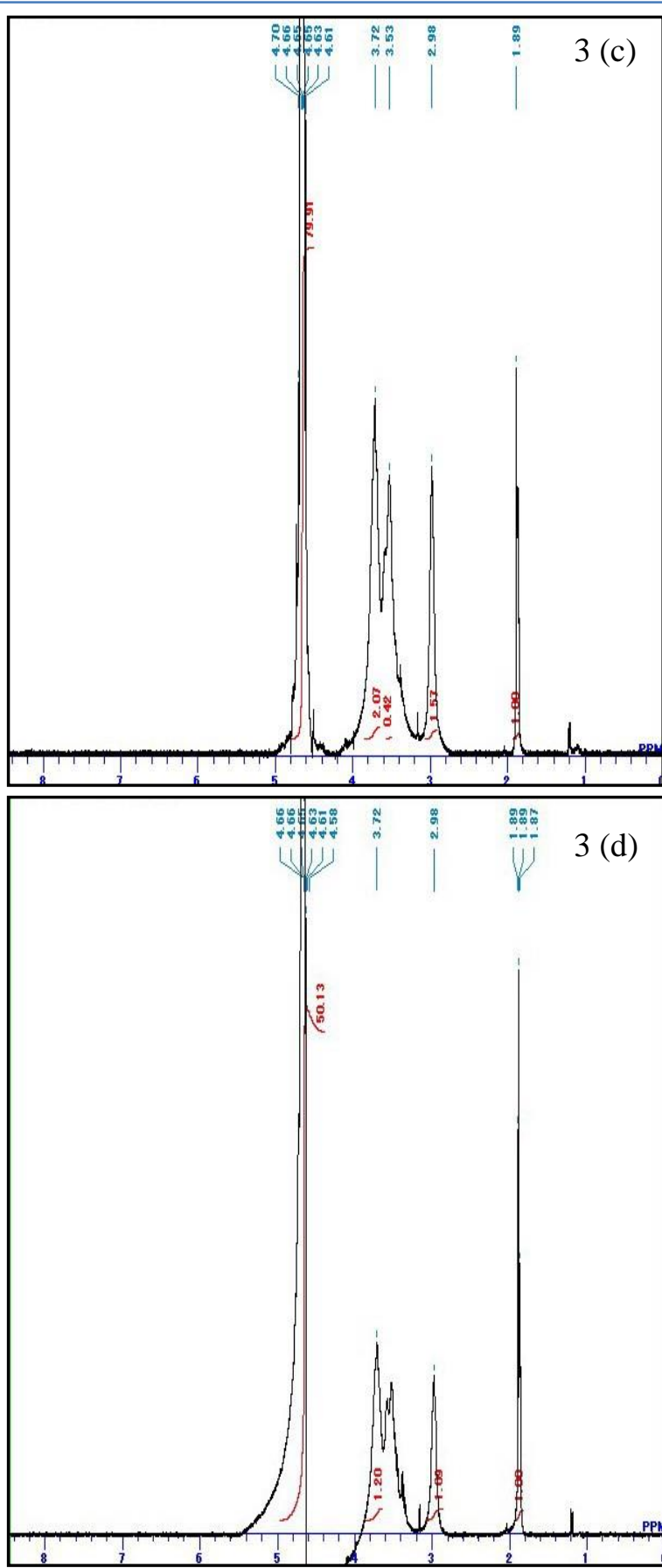

Figure 3: ${ }^{1} H$ NMR spectra of (a) chitosan powder and chitosan fibers after incubation of chitosan solution in open air at (b) 0 week (c) 2 weeks (d) 4 weeks

All 0 wk CTS, 2 wk CTS and 4 wk CTS fiber of ${ }^{1} \mathrm{H}$ NMR spectra were almost the same as original CTS powder spectrum. The chitosan signals of $\mathrm{H}$ 1 ( $\delta$ 4.7), $\mathrm{H}_{2}\left(\delta\right.$ 2.98), $\mathrm{H}_{3}, \mathrm{H}_{4}, \mathrm{H}_{5}(\delta$ 3.5-3.7), $\mathrm{NHCOCH}_{3}(\delta$ 1.89) ppm were qualified in all spectra. The formyl group at the signal of $\delta 8.1 \sim$ 8.4 in ${ }^{1} \mathrm{H}$ NMR was not occurred in all spectra of chitosan fibers [32]. The ${ }^{1} \mathrm{H}$ NMR data has also been confirmed that the character of original chitosan has not been changed under the aqueous formic acid with time. Moreover, there has no signal other than chitosan signals in all ${ }^{1} \mathrm{H}$ NMR spectra of fibers. According to the FTIR and ${ }^{1} \mathrm{HNM}$ data of chitosan samples, the character of chitosan fiber after spinning could observe the same nature as those of chitosan original. This fact can have proved that the chitosan in mild aqueous formic acid solution can be spun to get the fibers without losing the original characteristic of chitosan and also using sample coagulation bath $6 \mathrm{M} \mathrm{CaCl}_{2} \cdot 2 \mathrm{H}_{2} \mathrm{O}$ solution.

The surface and cross-sectional morphological structures can approve the statement of chitosan fibers. The surface morphology of chitosan fibers is shown in figure $4 \mathrm{a}, \mathrm{b}$, c. All fibers had been achieved with very smooth surface in morphology and rigid structure. The diameter of chitosan fibers was in the range of $50 \sim 63 \mu \mathrm{m}$. The image of cross-section of chitosan fiber (4wk CTS fiber) was shown in Figure 4. d, the chitosan fibers were obtained to have a rather round crosssection in all spinning and it was found to be nonporous structure. Other fibers were also observed the same morphology in the cross section.
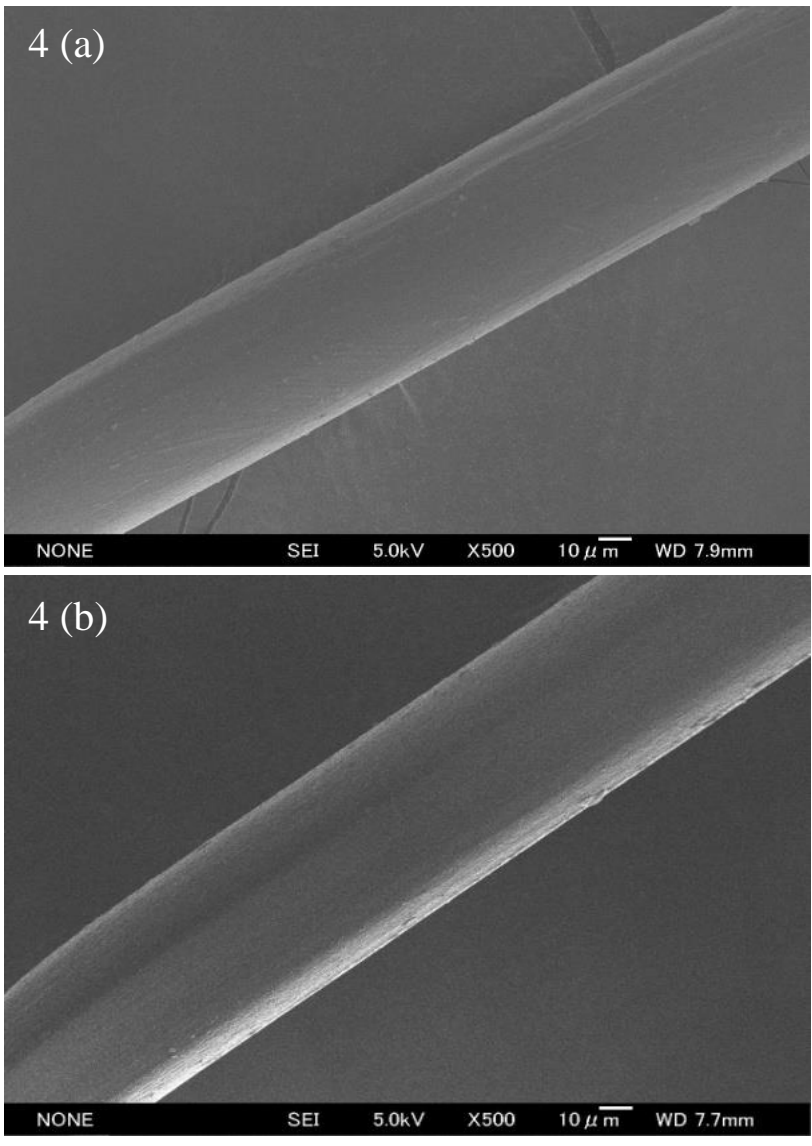
Spinning Process of Chitosan Fiber with Low Concentration of Formic Acid Solution and its Characteristics
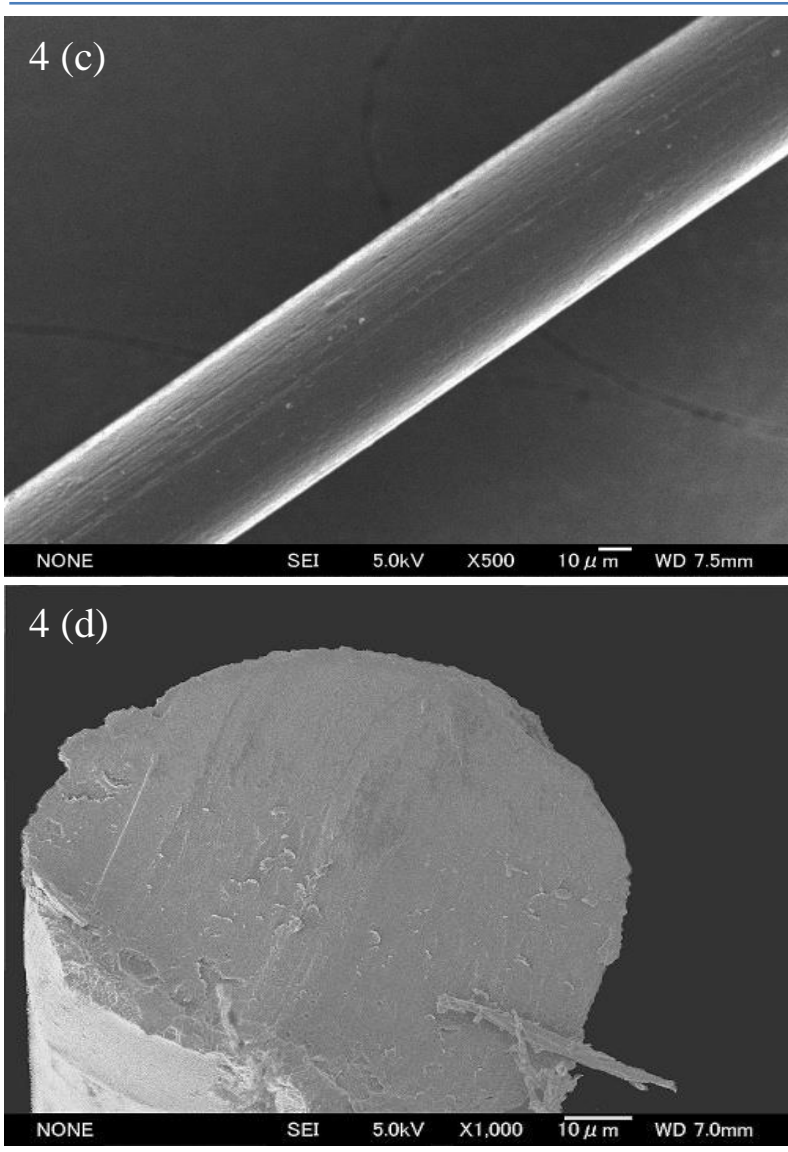

Figure 4: Representative SEM images of surface morphology of chitosan fibers after incubation of chitosan solution in open air ; (a) the 0 wk CTS fiber (b) the 2 wk CTS fiber (c) the 4 wk CTS fiber (d) cross-section of 4 wk CTS fiber

\subsection{Mechanical Properties of Chitosan Fiber}

The evaluation of tensile strength and knot strength of resulted chitosan fibers in dry and wet condition were carried out at $25^{\circ} \mathrm{C}$ according to the existing program of the apparatus ORIENTEC STA-1150 RTC. The force at breaking point of fiber was measured as tensile stress $(\mathrm{cN})$ that was transferred into tensile/knot strength (cN/dTex). Tensile and knot strengths of chitosan fibers in both dry and wet conditions are shown in Table 4. The chitosan fibers obtained from preparation of aqueous formic acid condition were resulted high tenacity (dTex $50,48 \& 46)$. According to the published reports, the tenacity of chitosan fibers was about dTex 3 $\sim 22$ when using the acetic acid as a solvent system for chitosan spinning. Tensile strength of 4 wk CTS fiber in dry state was $0.88 \mathrm{cN} / \mathrm{dTex}$, which are about two times higher than that of 0 wk CTS fiber in dry state $(0.59 \mathrm{cN} / \mathrm{dTex})$. Tensile strength of chitosan fibers was gradually increased when the time course of chitosan reaction with aqueous formic acid solution in open air was increased. Such improvement of the tensile strength might be caused by some interactions of $\mathrm{NH}_{3}{ }^{+}$groups of chitosan molecules with carboxylic groups of formic acid. These results showed almost the same strength value like the previous studies. Tensile strength of 0 wk CTS and 4 wk CTS fibers in the wet state were not much changed and knot strength in wet state of all fibers was found to be almost the same value. The knot strength of the chitosan fibers in 0 wk CTS and 2 wk CTS in dry state were $(0.389$ \& $0.66 \mathrm{cN} / \mathrm{dTex})$, that of $4 \mathrm{wk}$ CTS was $(0.76$ $\mathrm{cN} / \mathrm{d}$ Tex).

Table 4: Mechanical properties of chitosan fibers after incubation of chitosan solution at 0, 2 and 4 weeks in open air ( 0 wk CTS, 2 wk CTS and 4 wk CTS)

\begin{tabular}{|c|c|c|c|c|c|c|c|}
\hline \multirow{2}{*}{ Chitosan fiber } & \multirow{2}{*}{$\begin{array}{l}\text { Tenacity } \\
\text { dTex }\end{array}$} & \multicolumn{3}{|c|}{ Dry } & \multicolumn{3}{|c|}{ Wet } \\
\hline & & $\begin{array}{l}\text { Strength } \\
\text { cN/dTex }\end{array}$ & $\begin{array}{l}\text { Elongation } \\
\%\end{array}$ & $\begin{array}{l}\text { Stress } \\
\text { Mpa }\end{array}$ & $\begin{array}{l}\text { Strength } \\
\text { cN/dTex }\end{array}$ & $\begin{array}{l}\text { Elongation } \\
\%\end{array}$ & $\begin{array}{l}\text { Stress } \\
\text { Мpa }\end{array}$ \\
\hline $\begin{array}{r}0 \text { wk CTS Tensile } \\
\text { Knot }\end{array}$ & $\begin{array}{l}50 \\
50 \\
\end{array}$ & $\begin{array}{l}0.59 \\
0.389 \\
\end{array}$ & $\begin{array}{l}31.48 \\
38.5 \\
\end{array}$ & $\begin{array}{l}126 \\
81.5 \\
\end{array}$ & $\begin{array}{l}0.35 \\
0.306 \\
\end{array}$ & $\begin{array}{l}31.1 \\
22.3 \\
\end{array}$ & $\begin{array}{l}77.9 \\
69.9 \\
\end{array}$ \\
\hline $\begin{array}{r}2 \text { wk CTS Tensile } \\
\text { Knot }\end{array}$ & $\begin{array}{l}46 \\
46 \\
\end{array}$ & $\begin{array}{l}0.73 \\
0.66 \\
\end{array}$ & $\begin{array}{l}10.3 \\
17.9 \\
\end{array}$ & $\begin{array}{l}119.8 \\
145 \\
\end{array}$ & $\begin{array}{l}0.47 \\
0.32 \\
\end{array}$ & $\begin{array}{l}29.9 \\
9.0 \\
\end{array}$ & $\begin{array}{l}78.07 \\
56.2 \\
\end{array}$ \\
\hline $\begin{array}{r}\text { wk CTS Tensile } \\
\text { Knot } \\
\end{array}$ & $\begin{array}{l}48 \\
48 \\
\end{array}$ & $\begin{array}{l}0.88 \\
0.76 \\
\end{array}$ & $\begin{array}{l}4.2 \\
14.7 \\
\end{array}$ & $\begin{array}{l}124.6 \\
152 \\
\end{array}$ & $\begin{array}{l}0.57 \\
0.347 \\
\end{array}$ & $\begin{array}{l}24.97 \\
7.9 \\
\end{array}$ & $\begin{array}{l}104.4 \\
52.9 \\
\end{array}$ \\
\hline
\end{tabular}


So tensile and knot strength can also be concluded that the longer the time of spinning solution in open air, the higher the tensile and knot strength of fiber in dry state. However, the ratio in wet and dry state of tensile and knot strength, it was found to be the knot strength of 0 wk CTS was larger than in the tensile strength. The ratios of wet and dry strength are summarized in Figure 5.

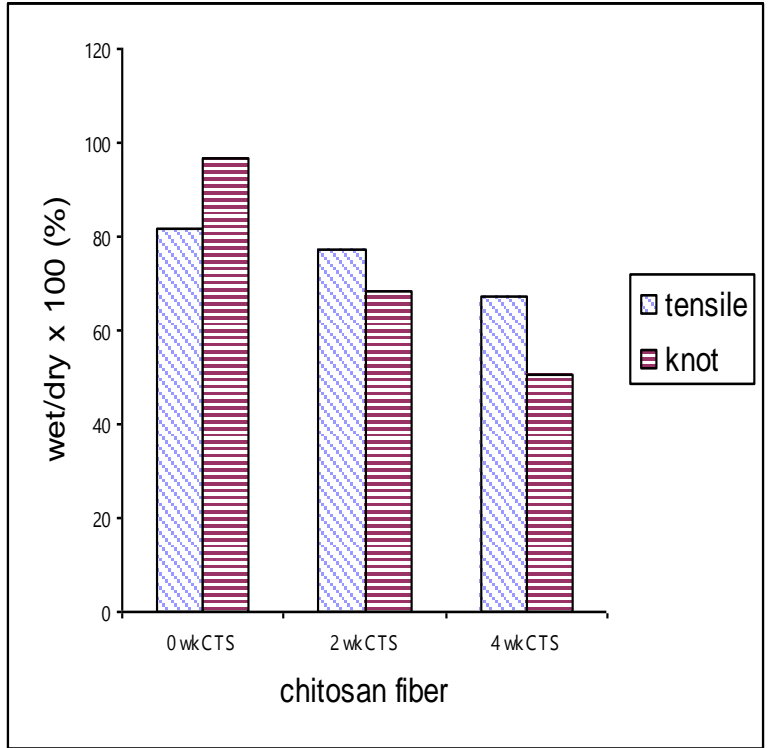

Figure 5: Wet-dry ratio of chitosan fibers after incubation of chitosan solution at 0, 2 and 4 weeks in open air (0 wk CTS, 2 wk CTS and 4 wk CTS)

The breaking elongation is also the most important qualified factor for fiber characteristic, which appears percentage in length when the fiber breaks due to a tensile force applied along the main fiber axis. The collecting data for breaking elongation of chitosan fiber is also shown in Table 4. The highest \% of breaking elongation in dry state could observe in $0 \mathrm{wk}$ CTS fiber and then it gradually decreased in 2 wk CTS fiber and $4 \mathrm{wk}$ CTS fiber. The highest tensile and knot stress were found in the 4 wk CTS fiber at dry state as 124.6 \& $152 \mathrm{Mpa}$, respectively. However, the $\%$ of breaking elongation decreased with increasing the time of spinning solution in open air. Based on these results, when the applied force was increased to break the axis of fiber; the breaking elongation was decreased significantly. Taken as a whole, the tenacity of chitosan fiber preparation with formic acid as higher than chitosan fiber using acetic acid.

\subsection{Crystallinity and Water Retention of Chitosan Fiber}

The crystallinity of chitosan fiber is shown in Figure 6 . The characteristic of anhydrous type chitosan crystalline structure is $\left(2 \theta=14.8^{\circ}(110)\right.$ and $\left.20=20^{\circ}(200)\right)$ [33]. The 0 wk CTS fiber was the characteristic for low crystalline structure; however, it has not seen amorphous chitosan form. The crystallinity of chitosan fibers in $2 \mathrm{wk}$ CTS and 4 wk CTS were gradually increased. The peak around $2 \theta=20^{\circ}$ being strongly increased at 4 wk CTS that showed extremely high crystallinity of chitosan molecules. So it can also be concluded that the crystallinity of chitosan fiber increased with increasing the duration of chitosan treated under aqueous formic acid in open air at room temperature.

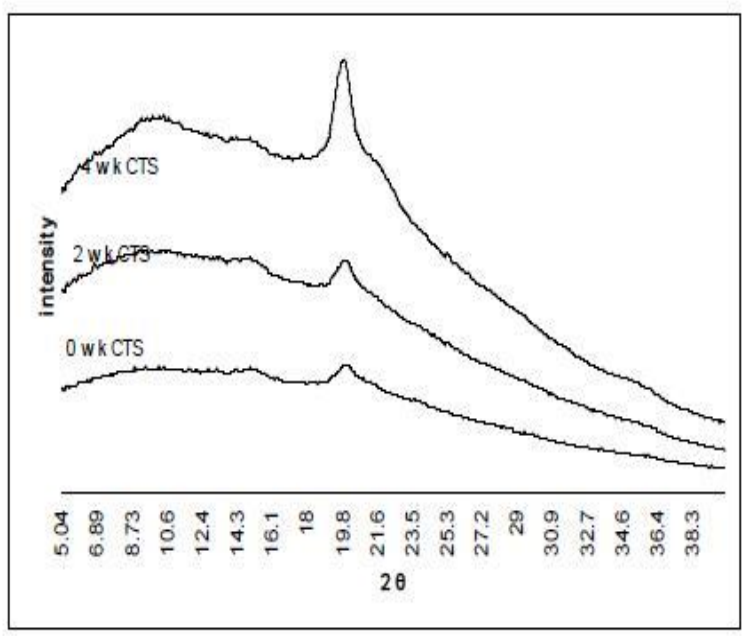

Figure 6: XRD pattern of chitosan fibers after incubation of chitosan solution in open air (O wk CTS, 2 wk CTS and 4 wk CTS)

The water retention percent of chitosan fibers are in the range of $138-333 \%$ (Table 3). The water retention value was dramatically increased as the reaction time of chitosan in aqueous formic acid in open air was increased; the lowest water retention property was found in the 0 wk CTS fiber and gradually increased on the 2 wk CTS and 4 wk CTS fibers. The 4 wk CTS fiber was the highest water retention value among of these chitosan fibers in this study. From this result, it can be concluded that the improvement of water 
Spinning Process of Chitosan Fiber with Low Concentration of Formic Acid Solution and its Characteristics

retention property depends on the matrix complexity of chitosan molecules in the fiber. Those may be different forms of chitosan chain matrix complexity in the fibers with time of spinning solution in open air even the molecular structure of chitosan was original nature after preparing the fiber. The higher crystallinity of chitosan fiber was obtained, the higher absorption probability due to recovery of hydrogen bonding in chitosan molecule that was confirmed in the Figure 5 and Table 3.

\subsection{Antimicrobial Activity}

In this study, the antimicrobial activity of chitosan fibers was investigated against the Escherichia coli and Styaphylococcus aureus; The reduction of microbial cell number (\%) was nearly the same value in 0 wk CTS, 2 wk CTS and 4 wk CTS fibers in E. coli and S. aureus. However the reduction number of $E$. coli on all chitosan
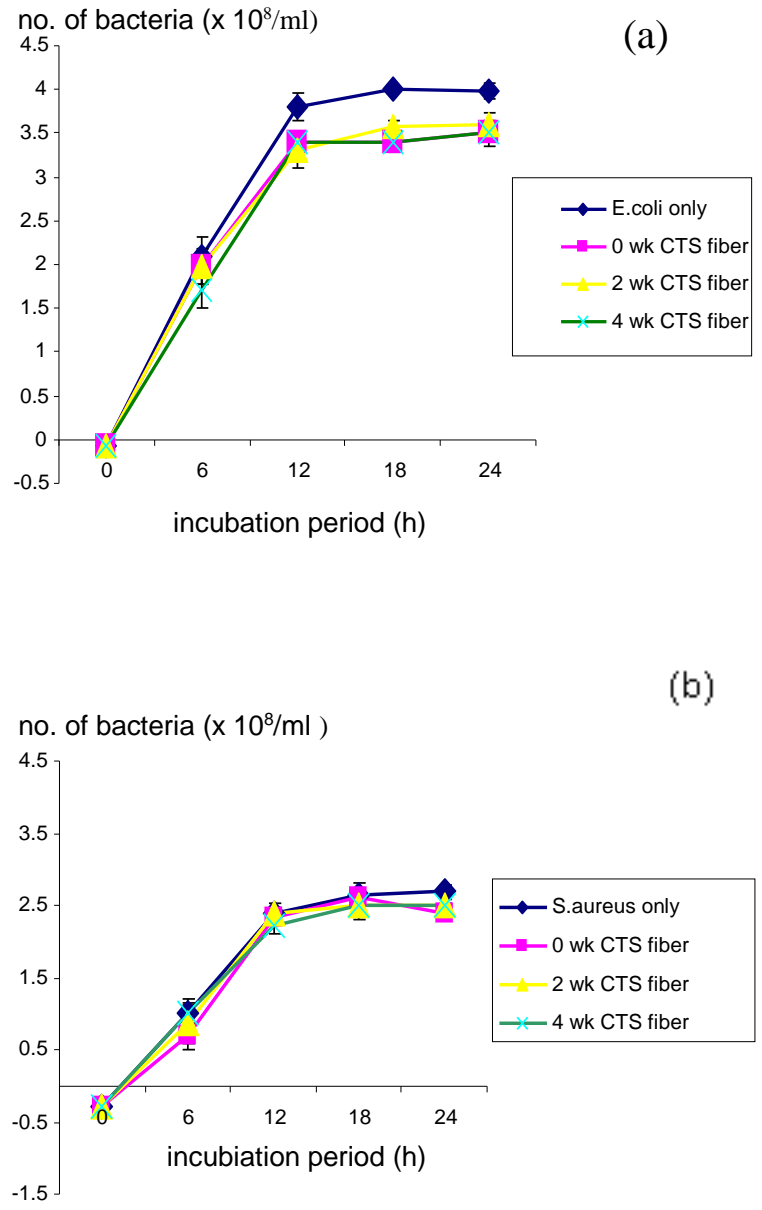

Figure 7: Antimicrobial activity of chitosan fibers after incubation of chitosan solution at 0, 2 and 4 weeks in open air ( 0 wk CTS, 2 wk CTS and 4 wk $C T S)$ (a) against E. coli (b) against $S$. aureus fibers were found to be no antimicrobial activities during $24 \mathrm{~h}$ as shown in Figure. 7. There are several investigations of antimicrobial activities in chitosan fiber by using low molecular weight and nano particles [34]-[36]. In this study, it has been used high molecular weight chitosan, so resulted fibers cannot afford antimicrobial activity to its environment using these two bacteria within 24 h.

\section{Conclusions}

The preparation of chitosan fiber under mild conditions by applying aqueous formic acid as a solvent has several advantages in chitosan spinning methods. Firstly, it could reduce the chitosan concentration (7\%) for fiber formation than previous method of Tamura et al., (2004) where $(10 \%)$ chitosan concentration was used. A modified coagulation system for chitosan fiber, aqueous $6 \mathrm{M} \mathrm{CaCl} 2.2 \mathrm{H}_{2} \mathrm{O}$ has also been achieved to make high tenacity chitosan fiber as a wet spinning. It can be reduced the calcium chloride concentration and methanol amount in coagulation system, from over-saturation to $6 \mathrm{M}$ concentration. This aqueous coagulation system was much milder spinning condition than that of chitosan dope in $10 \%$ acetic acid. The better spinability was found due to the addition of ethyl acetate to the spinning solution as a lubricant. The incubation time of chitosan spinning solution has been resulted the chitosan fibers without changing of original nature of chitosan in aqueous formic acid. The molecular characteristic of chitosan fibers has been confirmed by the data of FTIR and ${ }^{1}$ HNMR. The cross section of chitosan fiber by SEM confirmed the no porous in fiber. There was a certain point for each chitosan fiber and the strength of the chitosan fiber was gradually increased with an increasing the duration of chitosan in aqueous formic acid in open air at room temperature. The chitosan fiber demonstrated its better quality in spinning state, tenacity, breaking elongation, crystallinity and morphology. The water retention of chitosan fiber showed its high hydrophilicity so that chitosan fibers can be used for biomedical applications especially in wound dressing, textile fields for hospital applications and also in the field of waste management. 
Han et al., J. Mod. Mater.; Vol. 1, Issue 1, pp: 24-34, 2016

\section{Acknowledgements}

The authors thank to Prof. Seiichi Tokura for his valuable suggestions on the whole of this study and Director General Win Khaing Moe for his encouragement to fulfil of publication.

\section{How to Cite this Article:}

T. Han, N. Nwe, P. Win, and H. Tamura, "Spinning Process of Chitosan Fiber with Low Concentration of Formic Acid Solution and its Characteristics", J. Mod. Mater., vol. 1, no. 1, pp. 24-34, Jul. 2016. doi:10.21467/jmm.1.1.24-34

\section{References}

[1] S.B. Gudmund, A. Thorleif \& S. Paul, "Chitin and chitosan: Sources, chemistry, biochemistry, physical properties and applications", London, New York, Elisevier, 831,1989

[2] S.S. Koide, "Chitin-chitosan properties, benefits and risk", Nutrition Research, 18, 1091-1101, 1998.

[3] R.L. Whistler (ed.), "Polysaccharide chemistry", Academic Press, New York, 395,1983.

[4] M. Yalpani, F. Johnson \& L. E. Robinson, "Chitin, chitosan: Sources, chemistry, biochemistry, physical properties and applications", Amsterdam, Elsevier,1992.

[5] E.R. Pariser \& D.P. Lombadi, "Chitin source book", A guide to research literature, Wiley, 1980.

[6] H.W.T. Matthew \& V.M. Sundararajan, "Porous chitosan scaffolds for tissue engineering”, Biomaterial, 20, 11331142,1999 .

[7] R.A.A. Muzzarelli, "Chitin", Oxford Pergamon Press, 136,1977.

[8] G.A.F. Roberts "Chitin chemistry", London, (ed. Mac. Millan), 1992

[9] Q. Li, B. Song, Z. Yang \& H. Fan, "Electrolytic conductivity behaviors and solution conformations of chitosan in different acid solutions", Carbohydrate polymers, 63, 272-282, 2006.

[10] S. Tokura, S.I. Nishimura, N. Nishi, K. Nakamura, O. Hasegawa, H. Sashiwa \& H. Seo, "Preparation and some properties of variously deacetylated chitin fibers," Sen- $i$ Gakkaishi, 43, 288-293,1987.

[11] O. C. Agboh., \& Y. Qin, "Chitin and chitosan fibers", Polymer for advance technologies, 8, 355-365.1997.

[12] G.C. East \& Y. Qin," Wet spinning of chitosan and the acetylation of chitosan fibers." Journal of Applied Polymer Science, 50, 1773, 1993.

[13] Mitsubishi Rayon Co., Ltd, Japanese patent 81, 112, 937, 1980

[14] Mitsubishi Rayon Co., Ltd, Japanese patent 81, 106, 901, 1980.

[15] Fuji spinning Co.,Ltd. Japanese patent 59, 116, 418, 1984.

[16] United State patent 5897821, 1999.

[17] H. Tamura \& S. Tokura,"Preparation of chitosan coated alginate filament", Material Science and Engineering C, $20,143-147,2002$

[18] A. Watthanaphanit, P. Supaphol, T. Furuike, S. Tokura, H. Tamura \& R. Rujiravanit, "Novel chitosan-spotted alginate fibers from wet-spinning of alginate solutions containing emulsified chitosan-citrate complex and their characterization", Biomacromolecules, 10(2), 320-327, 2009.

[19] D.Yumin, F. Lihong, Z. Baozhong, Y. Jianhong, Z. Jinping, F.K. John, "Preparation and properties of alginate/carboxymethyl chitosan blend fibers", Carbohydrate Polymers, 65, 447-452,2006.

[20] K. Tuziakoglu, C.M. Alves, J.F. Mano \& R.L. Reis, "Production and characterization of chitosan fibers and 3D fiber mesh scaffolds for tissue engineering applications" Macromolecular Bioscience, 4, 811-819, 2004.
[21] S.H. Lee, S.Y. Park \& J.H. Choi, "Fiber formation and physical properties of chitosan fiber crosslinked by epichlorohydrin in a wet spinning system: The effect of the concentration of the crosslinking agent epichlorohydrin", Journal of Applied Polymer Science, 92, 2054-2062, 2004.

[22] H.Tamura, Y. Tsuruta, K. Itoyama, W. Worakitanchanakul, R. Rujiravanit \& S. Tokura, "Preparation of chitosan filament applying new coagulation system", Carbohydrate Polymers, 56, 205$211,2004$.

[23] H. Tamura, "Dissolution of chitin in calcium chloride solvent system", Polymer Preparation Japan, 55, 1862, 2006.

[24] H. Tamura, H. Nagahama and S. Tokura, "Preparation of chitin hydrogel under mild conditions", Cellulose, 13, 357-64, 2006.

[25] H.Tamura, H. Nagahama and S. Tokura., "Preparation of chitin hydrogel under mild conditions", Journal of Applied Polymer Science, 104, 3909-16, 2007.

[26] J.G. Domszy, G.A.F. Roberts, " Evaluation of infrared spectroscopic techniques for analyzing chitosan", Makromolecular Chemistry, 186,1671-1677, 1985.

[27] M. Miya, R. Iwamoto, S.Yoshikawam and I. Mina, "I.r spectroscopic determination of $\mathrm{CONH}$ content in highly deacetylated chitosan", International Journal of Biological Macromolecules, 2, 323-324, 1980.

[28] T. Sannan, K. Kurita, K. Ogura and Y. Iwakura, "Studies on chitin, I.r spectroscopic determination of degree of deacetylation", Polymer; 19, 458-459, 1987.

[29] S. Sabnis, L.H. Block, "Improved infrared spectroscopic method for the analysis of degree of $N$-acetylation of chitosan", Polymer Bulletin, 39, 67-71, 1997.

[30] A. Baxter, M. Dillon, K.D. Taylor, GAF, Roberts, “ Improved method for IR determination of the degree of $N$ acetylation of chitosan", International Journal of Biology and Macromolecule, 14, 166-169,1992.

[31] J. Brugnerotto, J. Lizardib, F.M. Goycoolea, W ArguellesMonal, J Desbrières, M Rinaudo, " An infrared investigation in relation with chitin and chitosan characterization", Polymer; 42, 3569-3580, 2001

[32] E.K. William, J.X. Chong \& W.P. Jay, "NMR identification of the formic acid-modified residue in Alzheimer's amyloid protein", Journal of Neurochemistry, 62, 349-354, 1994.

[33] T. Yui, K. Imada, K. Okuyama, Y. Obata, K. Suzuki \& K. Ogawa, "Molecular and crystal structure of the anhydrous form of chitosan", Macromoleacules, 27, 7601-7605, 1994.

[34] S.H. Lim, S.M. Hudson, "Synthesis and antimicrobial activity of a water-soluble chitosan derivative with a fiberreactive group", Carbohydrate Research, 339, 2, 313-319, Jan 2004.

[35] F. Lidija, R. Tijana, T. Tina, "Adsorption and antimicrobial activity of soluble and precipitated chitosan on cellulose viscose fibers", Journal of Engineered Fibers and Fibrics, 7, 1, 50-57, 2012.

[36] R. Saranya, V. Krishnaveni, "Study of antimicrobial activity of chitosan on lyocell and recycled polyester yarns", International Journal of Innovative Research in Science, Engineering and Technology, 3,2,9480-9486, Feb 2014.

Publish your research article in AIJR journals$\checkmark \quad$ Online Submission and Tracking $\checkmark$ Peer Reviewed

$\checkmark$ Rapid decision

$\checkmark$ Immediate Publication after acceptance

$\checkmark$ Articles freely available online

$\checkmark \quad$ Retain full copyright of your article.

Submit your article at journals.aijr.in 